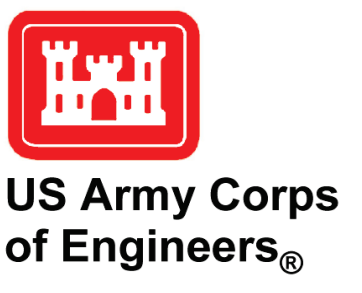

\section{Lock Operations Management Application (LOMA) Aid to Navigation (AtoN) Monitoring and Recovery (LAMaR)}

\title{
INTRODUCTION
}

Purpose. The purpose of this document is to specify the software requirements, architecture, and detailed design for the Lock Operations Management Application (LOMA) Aid to Navigation (AtoN) Monitoring and Recovery (LAMaR) application. This document is designed for the software developers maintaining LAMaR and is intended to aid these developers in understanding its architecture and underlying functionality.

Background. LOMA is a system designed to collect, store, and display operational data to assist lock operations. The primary data component is the Automatic Identification System (AIS) that provides real-time and historical display of commercial river traffic. The electronic AtoNs transmit information to the mariner to assist in navigation. This document is intended for the personnel involved in the development and maintenance of both LOMA and LAMaR.

\section{Pertinent Abbreviations}

- ACE-IT Army Corps of Engineers-Information Technology

- CHETN Coastal and Hydraulics Engineering Technical Note

- CHL Coastal and Hydraulics Laboratory, U.S. Army Engineer Research and Development Center (ERDC)

- DB Database

- DS DataSwitch

- IEEE Institute for Electrical and Electronics Engineers

- ITL Information Technology Laboratory, ERDC

- OS Operating System

- SMTP Simple Mail Transfer Protocol 
Scope. The boundary of this document is the custom software application known as LAMaR. While the remote AtoNs, DSs, Web Server, Web service, and ACE-IT SMTP are important components of the overall system, they are not considered to be components of LAMaR. They are considered to be components residing outside of LAMaR with which it must interface.

Overview. LAMaR will continually monitor and report the health of LOMA and attempt recovery of any AtoNs that are not functioning properly.

\section{SOFTWARE SPECIFIC REQUIREMENTS SPECIFICATION}

\section{Use Case Diagram}

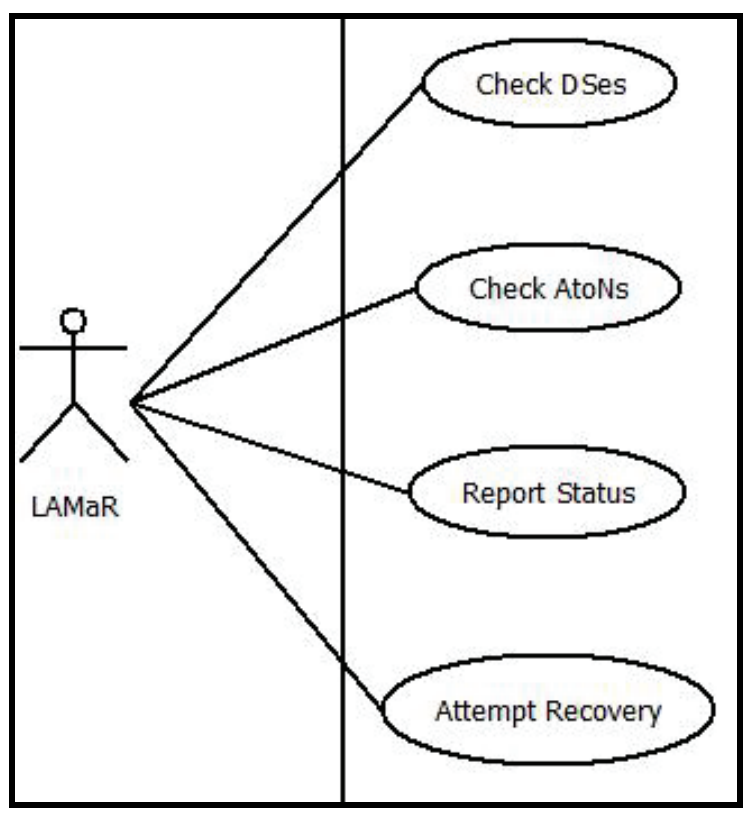

Details pertaining to the software specific requirements specification may be found in IEEE (1998).

\section{Use Cases}

\begin{tabular}{|l|l|}
\hline \multicolumn{2}{|c|}{ Check DSs } \\
\hline \hline Priority: & Required \\
\hline Description: & $\begin{array}{l}\text { Checks the status of the } \\
\text { DSs. }\end{array}$ \\
\hline Version(s): & All \\
\hline & $\begin{array}{l}\text { 1. } \begin{array}{l}\text { The application } \\
\text { checks the } \\
\text { connectivity status } \\
\text { of each DS. } \\
\text { Scenario: }\end{array} \\
\text { The application } \\
\text { records any DS with } \\
\text { no connectivity. }\end{array}$ \\
\hline
\end{tabular}

\begin{tabular}{|c|c|}
\hline \multicolumn{2}{|c|}{ Check AtoNs } \\
\hline Priority: & Required \\
\hline Description: & $\begin{array}{l}\text { Checks the status of the } \\
\text { AtoNs. }\end{array}$ \\
\hline Version(s): & All \\
\hline Scenario: & $\begin{array}{l}\text { 1. The application } \\
\text { checks the } \\
\text { connectivity status } \\
\text { of each AtoN. } \\
\text { 2. The application } \\
\text { records any AtoN } \\
\text { with no connectivity. }\end{array}$ \\
\hline
\end{tabular}




\begin{tabular}{|l|l|}
\hline \multicolumn{2}{|c|}{ Report Status } \\
\hline \hline Priority: & Required \\
\hline Description: & $\begin{array}{l}\text { Reports the status of the } \\
\text { system. }\end{array}$ \\
\hline Version(s): & All \\
\hline Scenario: & $\begin{array}{l}\text { The application reports } \\
\text { the overall system } \\
\text { status to the user(s). }\end{array}$ \\
& $\begin{array}{l}\text { 1. } \begin{array}{l}\text { The application is } \\
\text { unable to report the } \\
\text { overall system } \\
\text { status to the } \\
\text { user(s). } \\
\text { The application } \\
\text { records the overall } \\
\text { Scenario: }\end{array} \\
\text { system status. }\end{array}$ \\
\hline \multicolumn{2}{|l}{} \\
\hline
\end{tabular}

\begin{tabular}{|l|l|}
\hline \multicolumn{2}{|c|}{ Attempt Recovery } \\
\hline Priority: & Required \\
\hline Description: & $\begin{array}{l}\text { Attempts to recover } \\
\text { down AtoNs. }\end{array}$ \\
\hline Version(s): & All \\
\hline Scenario: & $\begin{array}{l}\text { 1. } \\
\text { The application } \\
\text { connects to each } \\
\text { down AtoN. } \\
\text { The application } \\
\text { resets each down } \\
\text { AtoN. }\end{array}$ \\
\hline Alternate & $\begin{array}{l}\text { 1. } \begin{array}{l}\text { The application is } \\
\text { unable to connect to } \\
\text { a down AtoN. } \\
\text { Scenario: }\end{array} \\
\text { The application } \\
\text { does not reset the } \\
\text { AtoN. }\end{array}$ \\
\hline \hline
\end{tabular}

\section{SOFTWARE ARCHITECTURAL DESIGN}

\section{Architecture}

Block Diagram. LAMaR will connect to the Web Services on the Web Server and each DS and determine the status of the DSs and AtoNs. After that, it will send a report of the overall system status to the user(s) via SMTP. Finally, the application will attempt to recover any down AtoNs.

\begin{tabular}{|c|c|}
\hline Name & Function \\
\hline AtoN & $\begin{array}{l}\text { The remote unit transmitting the information } \\
\text { to LOMA. }\end{array}$ \\
\hline DS & $\begin{array}{l}\text { The LOMA DS. On the DSs, it routes the } \\
\text { AtoN information to the LOMA DS on the } \\
\text { Web Server. On the Web Server, it routes } \\
\text { the information to the LOMA DB, the LOMA } \\
\text { Plotter, and the LOMA Web services. }\end{array}$ \\
\hline DS Server & The server on which the LOMA DS resides. \\
\hline LAMaR & $\begin{array}{l}\text { The application of which this document is } \\
\text { the focus. }\end{array}$ \\
\hline OS & The OS installed on the server. \\
\hline $\begin{array}{l}\text { SMTP } \\
\text { Server }\end{array}$ & $\begin{array}{l}\text { The SMTP server which is responsible for } \\
\text { transporting the e-mail correspondence. }\end{array}$ \\
\hline Web Server & $\begin{array}{l}\text { The server on which the primary LOMA DS } \\
\text { resides. }\end{array}$ \\
\hline
\end{tabular}

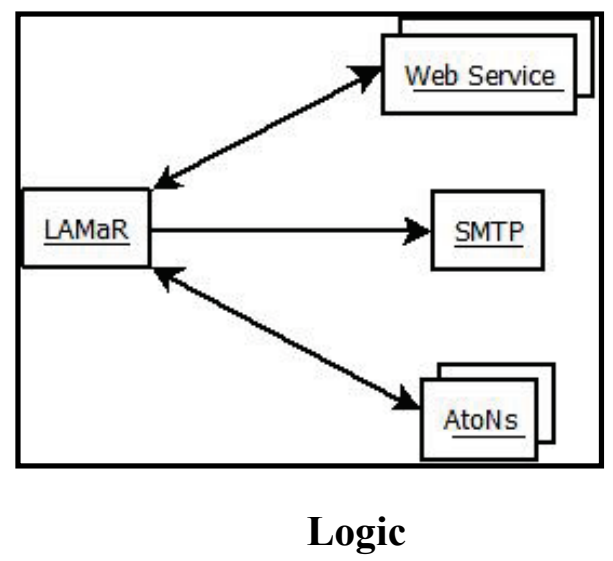

- Web Server

$\circ \mathrm{OS}$

- DS

- DS Server

○ OS

- DS

- LAMaR (on one DS only)

- SMTP Server

- AtoN 
ERDC/CHL CHETN-IX-50

August 2019

\section{Deployment Diagram}

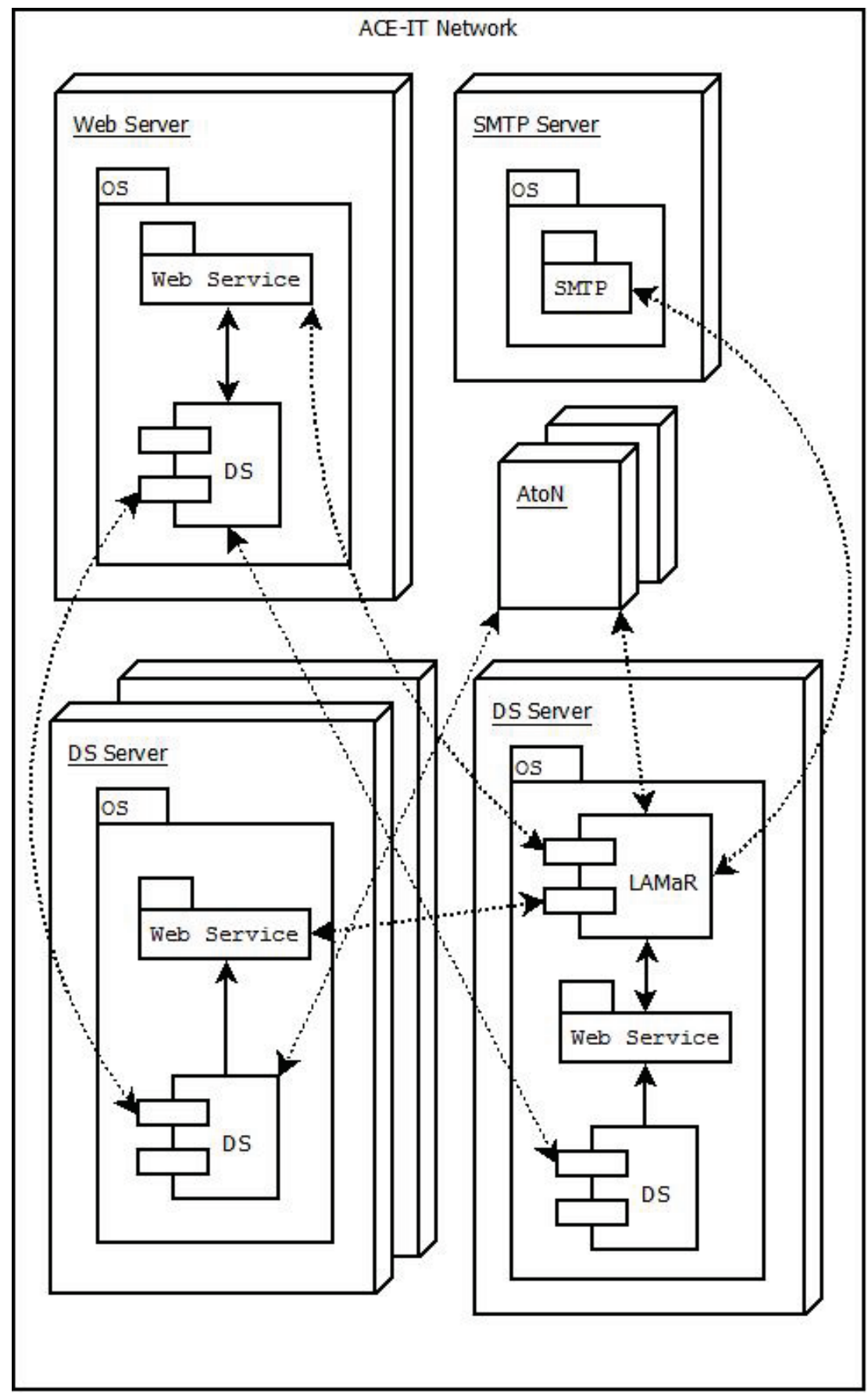

For simplicity, LAMaR will reside on one of the DSs. This will give the application ease of access to the Web service, SMTP server, and remote AtoNs as all the necessary rules and permissions will already be in place. 


\section{DETAILED SOFTWARE DESIGN}

\section{Structures}

- Properties

- Name: The name of the AtoN

- IP_Address: The IP address of the AtoN

- DataSwitch: The DS to which the AtoN communicates

- Constructors

○ AtoN (name, IP, DS)

- Parameters

- Name (the name of the AtoN)

- IP: (the IP address of the AtoN)

\begin{tabular}{|l|}
\hline \multicolumn{1}{|c|}{ AtoN } \\
\hline +Name: string \\
+IP_Address: string \\
+DataSwitch: string \\
\hline +AtoN (name: string, IP:string, DS:string) \\
\hline
\end{tabular}

- DS: (the DS to which the AtoN communicates)

\section{Private Methods}

/// 〈summary>

/// Configures the e-mail server settings based on the supplied configuration.

$/ / /</$ summary $>$

$/ / /\langle$ param name="configuration" $>$ The e-mail server configuration. $\langle/$ param $\rangle$

private static void Configure_Email_Server(Dictionary<string, List<object>> configuration)

/// <summary>

/// Retrieves the configuration from the configuration file

$/ / /</$ summary $>$

/// <param name="config_file_path" $>$ The path of the program configuration file. $</$ param $\rangle$

$/ / /<$ returns $>A$ map of the program configuration. $</$ returns $>$

private static Dictionary<string, List<object〉> Get_Configuration(string config_file_path)

$/ / /$ <summary >

/// Retrieves the DataSwitch statuses.

$/ / /</$ summary $>$

/// <param name="exclusions" $>$ The list of DataSwitch exclusions.</param $>$

/// <returns >A map of the DataSwitch statuses.</returns >

private static Dictionary<string, bool> Get_DataSwitch_Status(List<object> exclusions)

/// <summary>

/// Retrieves a map of down AtoNs and corresponding data.

/// </summary > 
/// <param name="DS" > The Web service client. </param>

/// <param name="DS_num" $>$ The DataSwitch number. $</$ param $>$

/// <param name="exclusions" $>$ The list of DataSwitch exclusions.</param >

private static void Get_Down_AtoNs

(

DSStatusServiceClient DS, string DS_num, List<object> exclusions

)

/// 〈summary>

/// Removes any AtoNs on the exclusion list.

/// </summary >

/// <param name="exclusions" $>$ The list of DataSwitch exclusions.</param>

private static void Remove_Excluded_AtoNs(List<object> exclusions)

/// <summary>

/// Composes and sends an e-mail to the administrator based on the supplied configuration and

/// generated body.

$/ / /</$ summary $>$

/// <param name="address" >The administrator e-mail address.</param>

/// <param name="subject" $>$ The e-mail subject. $</$ param $>$

/// <param name="body" $>$ The e-mail body. $</$ param $>$

private static void Send_Admin_Email(string address, string subject, string body)

// / summary>

/// Composes and sends an e-mail based on the supplied configuration and generated body.

/// </summary >

/// <param name="configuration" $>$ The e-mail configuration.$</$ param $>$

/// <param name="body" $>$ The body of the e-mail</param $>$

private static void Send_Email(Dictionary<string, List<object>> configuration, string body)

/// 〈summary >

/// Composes the body of the e-mail.

/// </summary $>$

/// <param name="signature" $>$ The e-mail signature. $\langle/$ param $\rangle$

/// <returns >The body of the e-mail.</returns>

private static string Write_Email_Body(string signature) 
FLOW: Logical diagrams for the LAMaR functions are provided below.

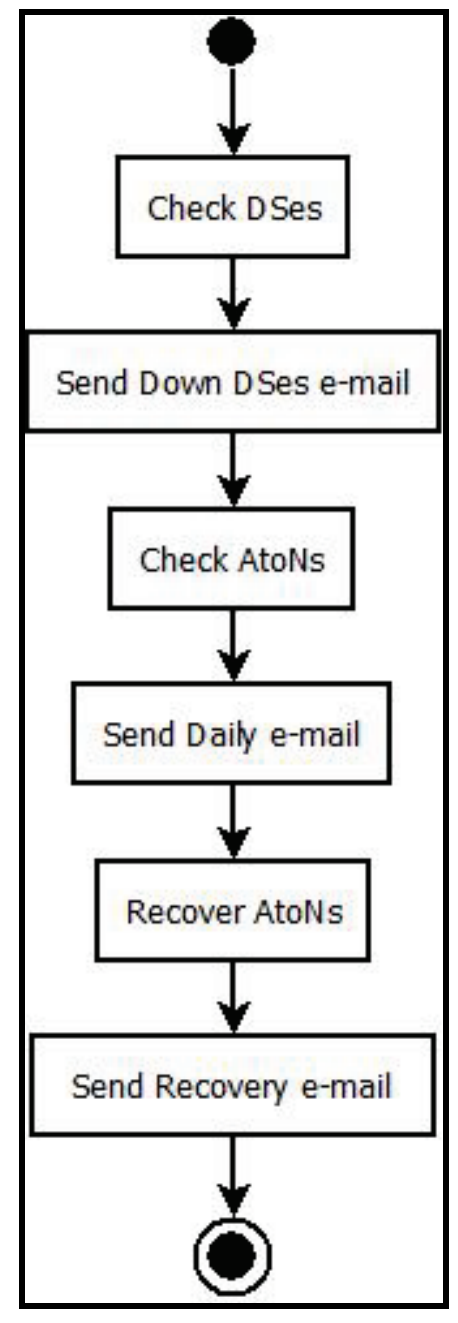

Program Flow

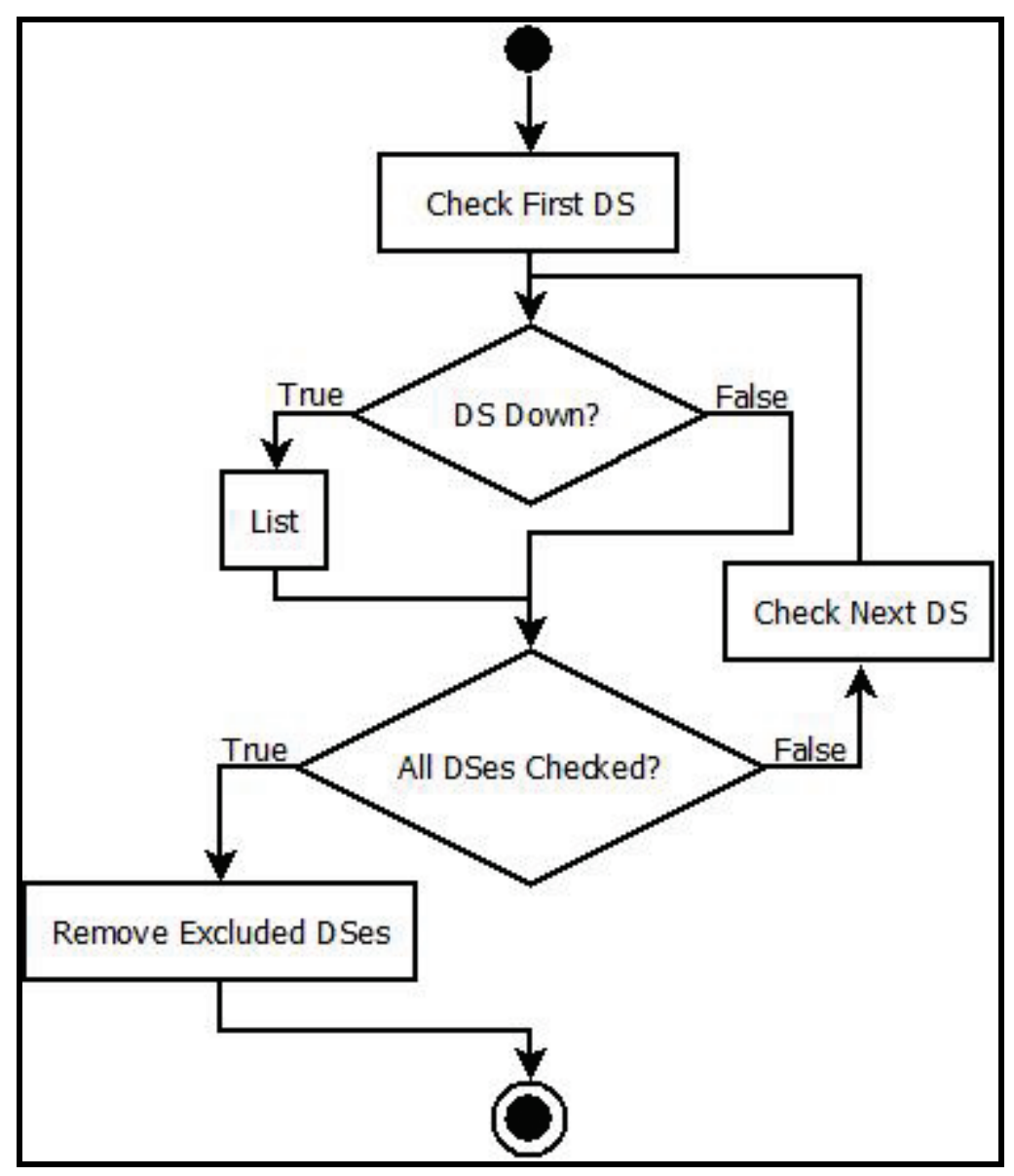

Check DSs. 


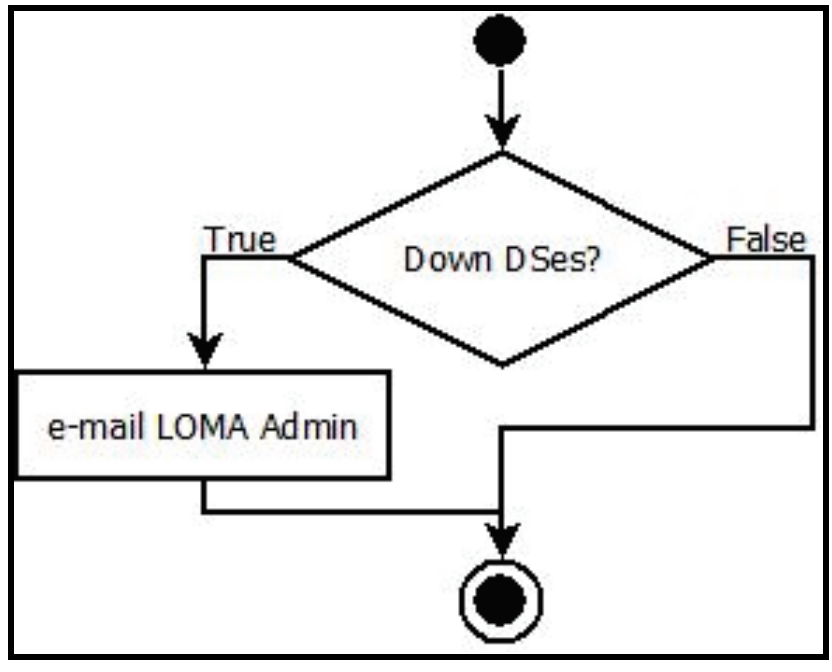

Send Down DSs e-mail.

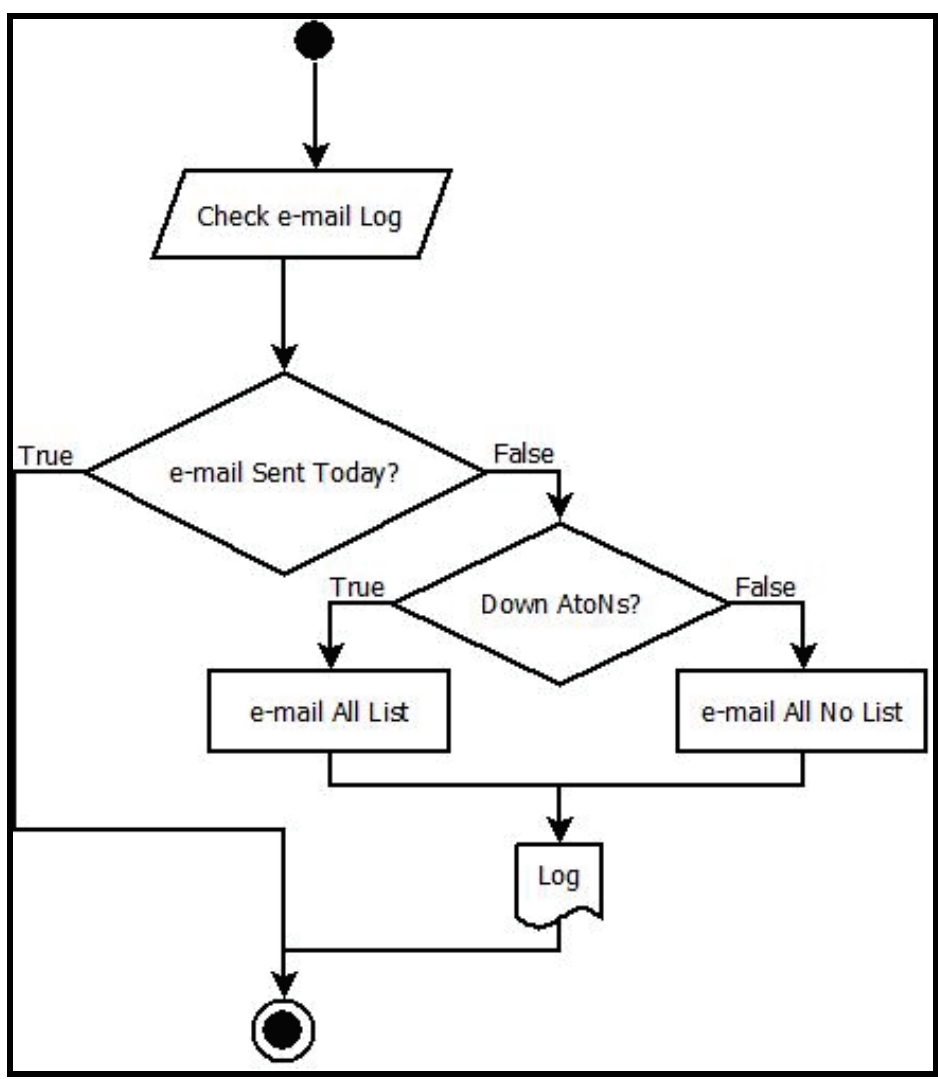

Send Daily e-mail.

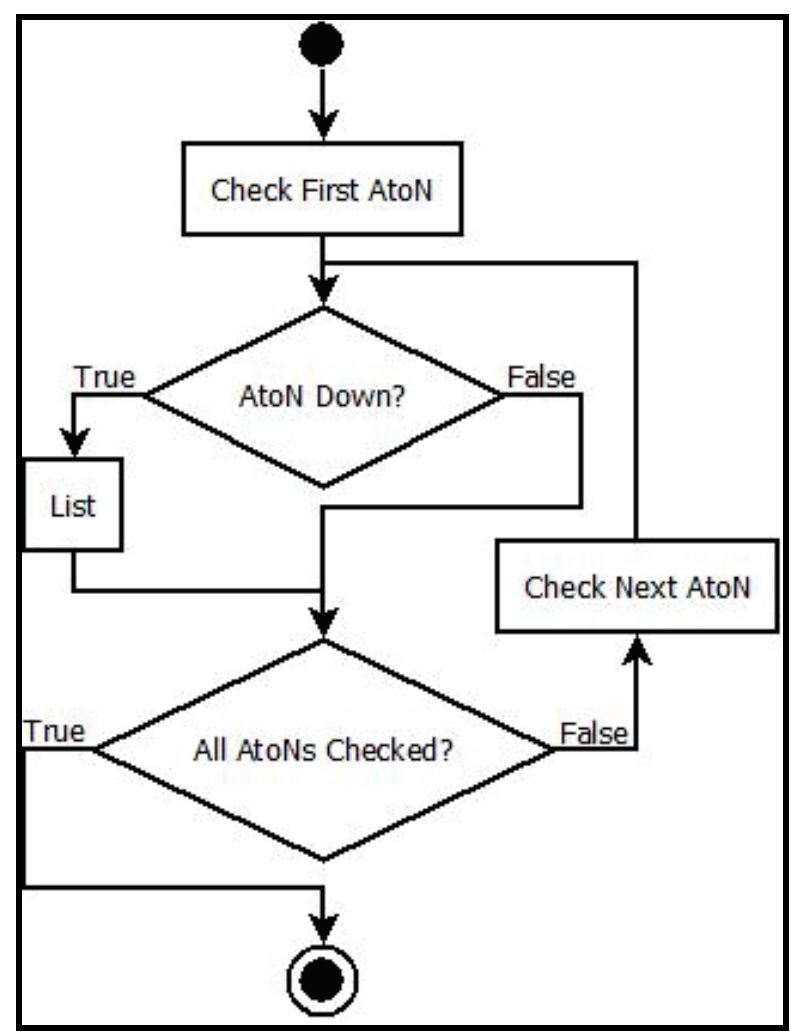

Check AtoNs. 


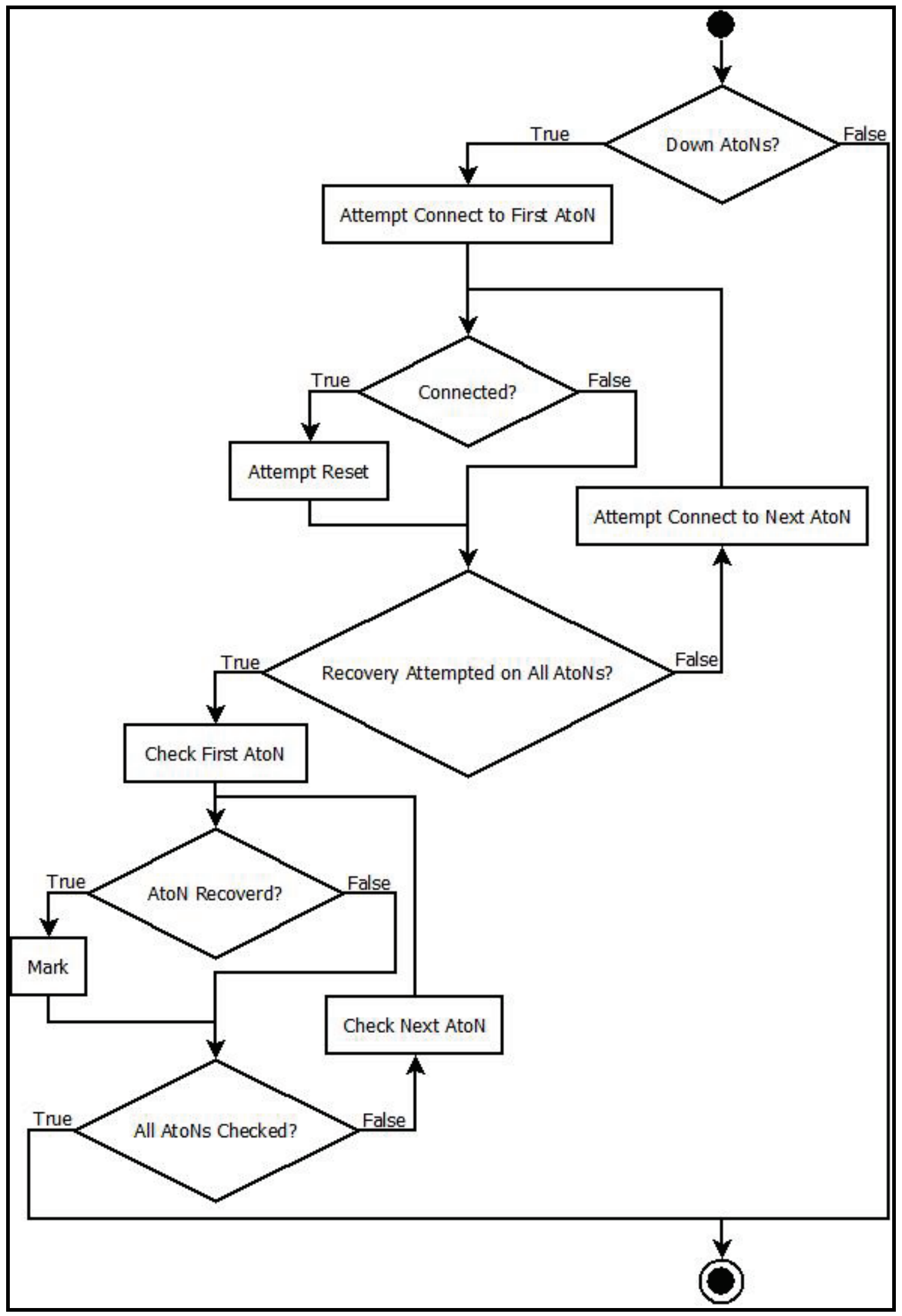

Recover AtoNs. 


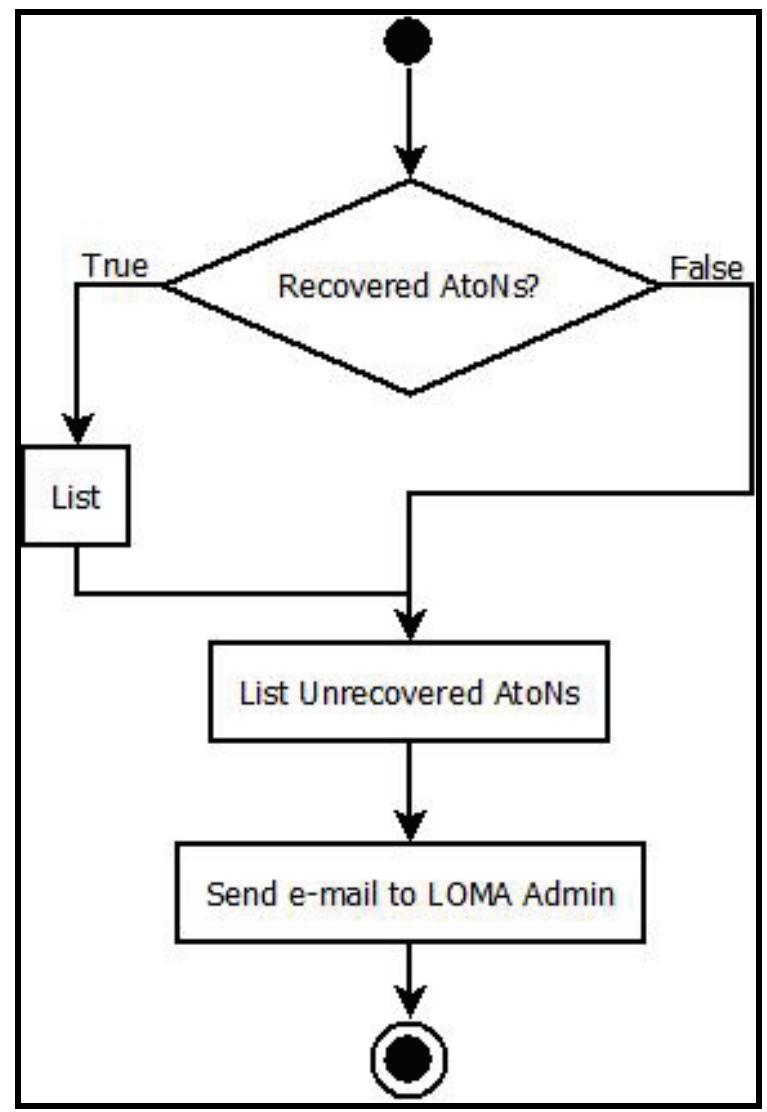

Send Recovery e-mail.

SUMMARY: LAMaR is a system developed to assist LOMA users in monitoring the operational status and identifying and recovering AtoNs that are not functioning properly.

ADDITIONAL INFORMATION: This CHETN was prepared as part of the USACE, ERDC, NavSys Research Program. The NavSys Program Manager was Charles E. Wiggins, ERDC CHL. Questions regarding this CHETN, LOMA, or LAMaR may be directed to the primary author and principal investigator Joseph H. Farrar, Computer Scientist, ERDC ITL Joseph.H.Farrar@usace.army.mil; or to other team members and co-authors Brian J. Tetreault, Navigation Systems Specialist (CHL) Brian.J.Tetreault@usace.army.mil; Michael F. Winkler, Research Hydraulic Engineer (CHL) Michael.F.Winkler@usace.army.mil; or James T. Kilroy, Navigation AIS Specialist (CHL) James.Kilroy@usace.army.mil. This CHETN should be referenced as follows:

Farrar, J. H., M. F. Winkler, and J. "Lee" Whitlow. 2019. Lock Operations Management Application (LOMA) Aid to Navigation (AtoN) Monitoring and Recovery (LAMaR). ERDC/CHL CHETN-IX-50. Vicksburg, MS: U.S. Army Engineer Research and Development Center. http://dx.doi.org/10.21079/ $\underline{11681 / 33629}$ 


\section{REFERENCES}

IEEE. 1998. Standard 830-1998; IEEE Recommended Practice for Software Requirements Specifications. Piscataway, NJ: Institute for Electrical and Electronics Engineers.

NOTE: The contents of this technical note are not to be used for advertising, publication, or promotional purposes. Citation of trade names does not constitute an official endorsement or approval of the use of such products. 